\title{
High expression of pituitary tumor-transforming gene-1 predicts poor prognosis in clear cell renal cell carcinoma
}

\author{
CAN WEI $^{1}$, XIAOLIANG YANG $^{1}$, JUNHUA XI $^{1}{ }^{1}$, WEI WU ${ }^{1}$, ZHENXING YANG $^{1}$, \\ WEI WANG ${ }^{1,2}$, ZHIGUO TANG ${ }^{1,2}$, QUANSHENG YING ${ }^{1,2}$ and YANBIN ZHANG ${ }^{1}$ \\ Departments of ${ }^{1}$ Urology and ${ }^{2}$ Pathology, Hefei Hospital Affiliated to Anhui Medical University/ \\ The Second People's Hospital of Hefei, Hefei, Anhui 230011, P.R. China
}

Received July 3, 2014; Accepted September 30, 2014

DOI: $10.3892 / \mathrm{mco} .2014 .478$

\begin{abstract}
Pituitary tumor-transforming gene-1 (PTTG1) is a recently identified oncogene involved in the progression of malignant tumors; however, the expression level of PTTG1 in clear cell renal cell carcinoma (ccRCC) and its potential value as a novel prognostic marker for ccRCC remains unclear. In this study, PTTG1 mRNA and protein levels were assessed in 44 paired ccRCC tissues and adjacent normal tissues by quantitative polymerase chain reaction (qPCR) and immunohistochemistry, respectively. Further immunohistochemical analysis was implemented in 192 samples of ccRCC to evaluate the associations between PTTG1 levels and the clinical characteristics in ccRCC. Reverse transcription qPCR and immunohistochemical analysis demonstrated that the PTTG1 mRNA and protein levels were significantly higher in ccRCC compared to normal tissues. In addition, the PTTG1 protein level in $192 \mathrm{ccRCC}$ samples was found to be significantly correlated with $\mathrm{T}$ stage, $\mathrm{N}$ classification, metastasis, recurrence and Fuhrman grade, whereas it was not associated with age and gender. Patients with low PTTG1 levels exhibited a better survival outcome compared to those with a higher PTTG1 level. PTTG1 expression and N stage were identified as independent prognostic factors for the overall survival of ccRCC patients. The results suggested that the overexpression of PTTG1 indicates a poor prognosis in ccRCC patients and, therefore, PTTG1 may serve as a novel prognostic marker for ccRCC.
\end{abstract}

\section{Introduction}

Renal cell carcinoma (RCC) is one of the leading causes of urological cancer-related mortality and clear cell renal cell carcinoma (ccRCC) is the most common type of RCC,

Correspondence to: Dr Yanbin Zhang, Department of Urology, Hefei Hospital Affiliated to Anhui Medical University/The Second People's Hospital of Hefei, Guangde Road, Hefei, Anhui 230011, P.R. China

E-mail: zhangyanbin1967@sina.com

Key words: clear cell renal cell carcinoma, pituitary tumor transforming gene-1, oncogene, prognosis accounting for $82 \%$ of RCC cases (1). Early-stage ccRCC is usually asymptomatic and difficult to accurately diagnose; in addition, ccRCC is resistant to conventional chemotherapeutic drugs and the overall clinical outcome is poor (2). The treatment of advanced ccRCC remains a major challenge for clinicians and ccRCC is responsible for $\sim 35 \%$ of RCC-related mortality cases (1). Thus, there is an urgent need for diagnostic and prognostic biomarkers in ccRCC. However, there are currently no biomarkers for ccRCC in routine clinical practice. Over the last few years, prognostic markers for ccRCC, such as senescence-associated protein p400 (3), glyoxalase-1 (4), transforming growth factor- $\beta$-activated kinase-1 (5) and serum miR-210 (6), have emerged; however, their large-scale clinical application is not feasible. Therefore, novel diagnostic and prognostic markers of ccRCC would be valuable in high-risk individuals and in those with existing disease.

Pituitary tumor-transforming gene-1 (PTTG1) was first isolated from rat pituitary tumor cells in 1997 and was identified as an oncogene, as PTTG1 overexpression was found to induce cellular transformation in vitro and tumor formation in nude mice (7). As a human securin, PTTG1 is involved in the mitotic spindle checkpoint pathway and inhibits sister chromatid separation to ensure chromosomal stability $(7,8)$. In contrast to its restricted expression in normal tissues, PTTG1 is abundantly detected in a wide variety of tumors and is associated with metastasis and poor clinical outcome, suggesting that PTTG1 may play a role in tumorigenesis $(9,10)$. A higher PTTG1 expression is observed in cancer cells compared to that in adjacent normal cells, although the underlying mechanisms has not been fully elucidated. Moreover, the association between PTTG1 expression and prognosis remains ambiguous. In this study, we aimed to investigate the expression and clinical significance of PTTG1 in ccRCC and assess the association between PTTG1 expression level and prognosis.

\section{Materials and methods}

Patients and tissue samples. The 44 paired samples of ccRCC and normal adjacent tissues (ADTs) were collected from patients who underwent radical nephrectomy at the Department of Urology, Hefei Hospital Affiliated to Anhui Medical University, between November, 2007 and December, 2012. The ADT samples were collected at a distance of $2.0 \mathrm{~cm}$ from 
Table I. Association of pituitary tumor-transforming gene-1 (PTTG1) expression level with clinicopathological characteristics in clear cell renal cell carcinoma patients.

\begin{tabular}{|c|c|c|c|c|c|}
\hline \multirow[b]{2}{*}{ Parameters } & \multirow[b]{2}{*}{$\begin{array}{l}\text { Total no. } \\
(\mathrm{n}=192)\end{array}$} & \multicolumn{3}{|c|}{ PTTG1 expression } & \multirow[b]{2}{*}{ P-value } \\
\hline & & $\begin{array}{c}\operatorname{High}(+) \\
(\mathrm{n}=113)\end{array}$ & $\begin{array}{c}\text { Low (-) } \\
(\mathrm{n}=79)\end{array}$ & $\chi^{2}$ & \\
\hline Gender & & & & 1.819 & 0.177 \\
\hline Male & 108 & 59 & 49 & & \\
\hline Female & 84 & 54 & 30 & & \\
\hline Age (years) & & & & 0.560 & 0.454 \\
\hline$\geq 50$ & 125 & 76 & 49 & & \\
\hline$<50$ & 67 & 37 & 30 & & \\
\hline T stage & & & & 10.816 & 0.004 \\
\hline $\mathrm{T} 1$ & 97 & 47 & 50 & & \\
\hline $\mathrm{T} 2$ & 51 & 32 & 19 & & \\
\hline $\mathrm{T} 3 / 4$ & 44 & 34 & 10 & & \\
\hline $\mathrm{N}$ stage & & & & 4.674 & 0.031 \\
\hline No & 145 & 79 & 66 & & \\
\hline $\mathrm{N}+$ & 47 & 34 & 13 & & \\
\hline Metastasis & & & & 4.169 & 0.041 \\
\hline No (M0) & 130 & 70 & 60 & & \\
\hline Yes (M1) & 62 & 43 & 19 & & \\
\hline Recurrence & & & & 7.903 & 0.005 \\
\hline No & 157 & 85 & 72 & & \\
\hline Yes & 35 & 28 & 7 & & \\
\hline Fuhrman grade & & & & 14.719 & 0.002 \\
\hline $\mathrm{F} 1$ & 111 & 53 & 48 & & \\
\hline $\mathrm{F} 2$ & 51 & 26 & 25 & & \\
\hline F3 & 27 & 22 & 5 & & \\
\hline $\mathrm{F} 4$ & 13 & 12 & 1 & & \\
\hline
\end{tabular}

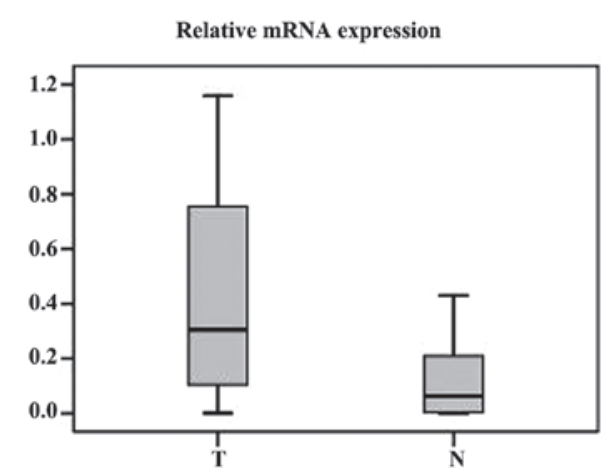

Figure 1. Reverse transcription-quantitative polymerase chain reaction analysis of pituitary tumor-transforming gene-1 (PTTG1) mRNA. The relative expression of PTTG1 mRNA in clear cell renal cell tumor (T) tissue samples was higher compared to that in the paired adjacent normal tissues $(N)(n=44$, $\mathrm{P}<0.001$ ). Bottom and top of the box, 25 th and the 75 th percentile, respectively; band near the middle of the box, 50th percentile (median); ends of the whiskers, 2.5 th and 97.5 th percentiles.

visible ccRCC lesions and were all properly maintained until reverse transcription-quantitative polymerase chain reaction (RT-qPCR) analysis. For immunohistochemical analysis of the PTTG1 protein, a total of 192 paraffin-embedded pathologically verified ccRCC samples were collected. All the patients had undergone radical nephrectomy performed at the Department of Urology, Hefei Hospital Affiliated to Anhui Medical University, between 2000 and 2013. The histological and clinical diagnoses of the tumors in all the patients were performed by the Department of Pathology in the same hospital. The characteristics of the 192 patients are summarized in Table I. Patient survival data were obtained via telephonical communication and data on clinical characteristics were obtained from the medical records. Tumor stage was reclassfied based on the 2011 Union for International Cancer Control TNM classification of malignant tumors and nuclear grading was performed according to the Fuhrman classification (11).

This study was approved by the Ethics Committee of Anhui Medical University and all the patients provided written informed consent.

$R T-q P C R$. RT-qPCR was performed as previously described (12). The corresponding primer sequences were as follows: PTTG1: sense primer, 5'-AAAGCTCTGTTCCTG CCTCA-3'; and reverse primer, 5'-GAGAGGCACTCC ACTCAAGG-3'. GAPDH: sense primer, 5'-GGAGTCCAC TGGCGTCTTCACC-3'; and reverse primer, 5'-GAGGAG TGGG TGTCGCTGTTG-3'. The relative expression levels of 


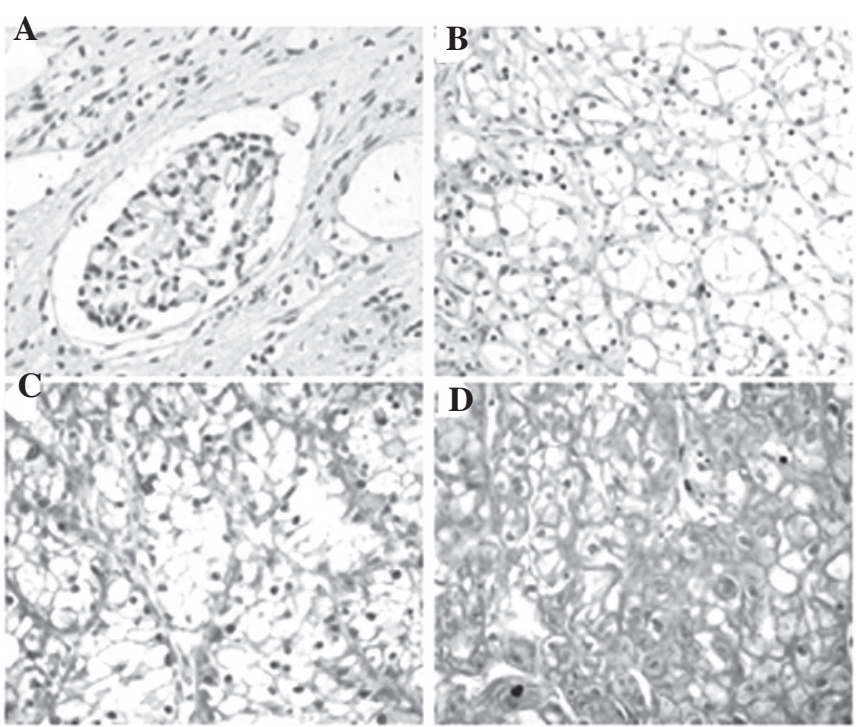

Figure 2. Immunohistochemical analysis of the expression of pituitary tumor-transforming gene-1 (PTTG1) protein. PTTG1 is mainly localized within the nuclei and cytoplasm. (A) Immunostaining of the adjacent normal tissue samples was negative or very weak (magnification, $x 400$ ). (B) Weak PTTG1 staining in cancerous tissue (magnification, $x 400$ ). (C) Moderate PTTG1 staining in cancerous tissue (magnification, $x 400$ ). (D) Strong PTTG1 staining in the majority of the tumor cells (magnification, $\mathrm{x} 400$ ).

PTTG1 were normalized to the geometric mean of GAPDH (internal control gene). The data were analysed via the comparative threshold cycle method (13).

Immunohistochemical analysis. Immunohistochemistry was performed to investigate PTTG1 expression in the 44 paired ccRCC and normal tissue samples. This was also implemented in the 192 ccRCC samples. All the procedures were performed as previously described (12). The sections were incubated with the monoclonal rabbit anti-human PTTG1 antibody (cat. no. sc-5843, dilution 1:400; Abcam, Cambridge, MA, USA).

Staining evalvation. The stained sections were reviewed by two independent pathologists. Scoring was based mainly on color intensity and extent. The proportion of cells expressing PTTG1 varied between 0 and $100 \%$ and the intensity of staining ranged from weak to strong. The proportion of PTTG1-expressing tumour cells was scored at low magnification on a scale of $0-5$ ( 0 , no positive cells; $1,0-5 \% ; 2,6-25 \% ; 3,26-50 \% ; 4,51-75 \%$; and $5,76-100 \%$ positive cells). The intensity score was determined at high magnification on a scale of 0-3 (0, negative; 1 , weakly positive; 2 , moderately positive; and 3 , strongly positive staining) and the final score was calculated by multiplying the two parameters, with scores of $0,1,2,3,4,5,6,8,9,10$, 12 and 15. The optimal cut-off values for PTTG1 levels were set by measuring the heterogeneity in overall survival rates using the log-rank test method. Low expression was defined as a total score of $<5$ and high expression as a total score of $\geq 5$. Thus, the stained sections were divided into two different groups by PTTG1 expression level.

Statistical analysis. Paired-sample t-tests were used in the RT-qPCR and immunohistochemical assays to analyze the significance of the differences in mRNA and protein expression level between ccRCCs and the ADTs. The correlation of PTTG1 expression with clinicopathological characteristics was assessed by the $\chi^{2}$ test. Survival curves were plotted according to the Kaplan-Meier method and compared by the log-rank test. A multivariate analysis according to Cox's proportional hazards regression model adjusted for clinicopathological factors (age, gender, tumor size, Fuhrman grade, TNM stage and PTTG1 expression) was performed to identify the clinical variables that were independently correlated with overall survival. The statistical analysis was performed using the SPSS 17.0 package (SPSS Inc., Chicago, IL, USA) and $\mathrm{P}<0.05$ was considered to indicate a statistically significant difference.

\section{Results}

RT-qPCR analysis of PTTG1 mRNA in 44 ccRCC tumor samples. RT-qPCR was performed to measure the expression of PTTG1 mRNA in 44 ccRCC tumor tissues and normal ADTs. Compared to normal tissues, 37 ccRCC tumor tissue samples exhibited a higher expression at the mRNA level (paired-sample t-test, $\mathrm{P}<0.001$; Fig. 1).

Immunohistochemistry analysis of PTTG1 protein expression in 44 ccRCC and paired ADT samples. Immunohistochemistry was applied to assess the expression and subcellular localization of the PTTG1 protein in 44 paraffin-embedded ccRCC and paired normal ADT samples. PTTG1 staining was present mainly in the nuclei and cytoplasm (Fig. 2). In normal renal tissue, PTTG1 protein expression was negative (29/44, score $=0)$ or low $(15 / 44$, score $\leq 5)$. The PTTG1 protein expression in the 44 tumor tissue samples was higher compared to that in the paired ADTs (paired-sample t-test, $\mathrm{P}<0.001$; Fig. 3 ).

Immunohistochemical analysis of the association between PTTG1 protein expression and clinical characteristics in 192 ccRCC samples. To further assess the correlation between PTTG1 expression and various clinicopathological parameters, a further immunohistochemical analysis was performed in 192 ccRCC samples. As shown in Table I, low expression of PTTG1 (score $\leq 4)$ was exhibited by 79 of the 192 tumor samples, whereas high expression (score $\geq 5$ ) was exhibited by 113 samples. Increased expression of PTTG1 in tumor samples was correlated with $\mathrm{T}$ stage $\left(\chi^{2}=10.816, \mathrm{P}=0.004\right)$, $\mathrm{N}$ classification $\left(\chi^{2}=4.674, \mathrm{P}=0.031\right)$, metastasis $\left(\chi^{2}=4.169\right.$, $\mathrm{P}=0.041)$, recurrence $\left(\chi^{2}=7.903, \mathrm{P}=0.005\right)$ and Fuhrman grade $\left(\chi^{2}=14.719, \mathrm{P}=0.002\right)$, while associations with age $\left(\chi^{2}=0.560\right.$, $\mathrm{P}=0.454)$ and gender $\left(\chi^{2}=1.819, \mathrm{P}=0.177\right)$ were not identified. High expression of PTTG1 was observed in 69.5, 62.7 and $77.3 \%$ of stage $\mathrm{T} 1, \mathrm{~T} 2$ and $\mathrm{T} 3 / 4$ ccRCC cases, respectively $\left(\mathrm{P}=0.004, \chi^{2}\right.$ test). High expression of PTTG1 was observed in 69.4 and $53.8 \%$ of stage N0 and N1/2 ccRCC cases, respectively $\left(\mathrm{P}=0.041, \chi^{2}\right.$ test $)$. High expression of PTTG1 was observed in 74.2 and $54.6 \%$ of ccRCC cases with and without metastasis, respectively $\left(\mathrm{P}=0.009, \chi^{2}\right.$ test). Finally, high expression of PTTG1 protein was observed in 80.0 and $54.1 \%$ of ccRCC cases with and without recurrence, respectively $(\mathrm{P}=0.005$, $\chi^{2}$ test).

Survival analysis. To further investigate the prognostic value of PTTG1 expression in ccRCC, Kaplan-Meier analysis 


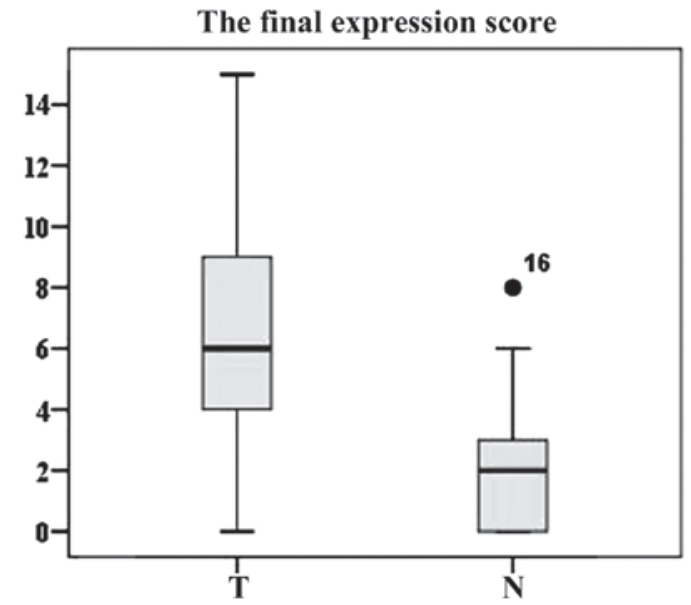

Figure 3. Increased protein expression of pituitary tumor-transforming gene-1 (PTTG1) in clear cell renal cell carcinoma (ccRCC). The relative protein expression of PTTG1 in ccRCC tissue samples was higher compared to that in the paired adjacent normal tissue samples $(n=44, P<0.001)$. Bottom and top of the box, lower and upper quartiles, respectively; band near the middle of the box, median; ends of the whiskers, 2.5 th and the 97.5 th percentiles. The four black dots represent the special value outliers. PTTG1+, higher level of PTTG1 expression; PTTG1-, lower level of PTTG1 expression.

$\mathbf{A}$

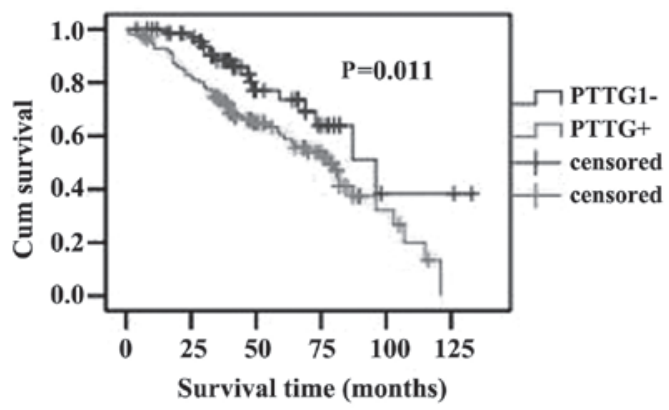

B

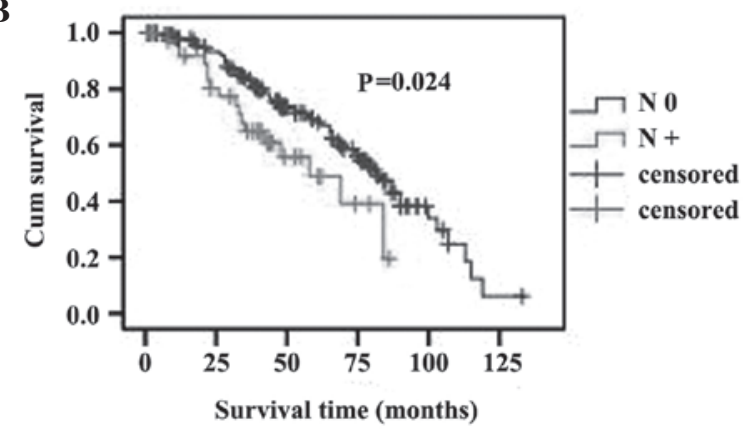

Figure 4. Overall survival analysis. (A) Survival analysis of primary clear cell renal cell carcinoma (ccRCC) patients $(n=192)$. Kaplan-Meier survival analysis of primary ccRCC patients following surgical resection with high pituitary tumor-transforming gene-1 (PTTG1+) $(n=113)$ and low PTTG1 (PTTG1-) ( $n=79)$ expression. The cumulative survival rate of patients in the PTTG1-high group was significantly lower compared to that of patients in the PTTG1-low or -negative group ( $\log$-rank, $\mathrm{P}=0.011)$. (B) Patients with no regional lymph node involvement (N0) exhibited a better prognosis compared to those with regional lymph node involvement $(\mathrm{N}+)(\log -\mathrm{rank}, \mathrm{P}=0.024)$.

and the log-rank test were applied to assess the association between PTTG1 expression level in ccRCC and prognosis. The level of PTTG1 expression was found to be correlated with the overall survival of ccRCC patients. Patients with higher PTTG1 expression (PTTG1+) exhibited poorer survival rates
Table II. Multivariate Cox regression analysis for the overall survival rates of clear cell renal cell carcinoma patients.

\begin{tabular}{lccc}
\hline Risk factors & $\begin{array}{c}\text { Relative } \\
\text { risk }\end{array}$ & $\begin{array}{c}95 \% \text { confidence } \\
\text { interval }\end{array}$ & P-value \\
\hline T stage & 3.001 & $0.366-1.970$ & 0.201 \\
M stage & 1.827 & $0.819-3.406$ & 0.077 \\
N stage & 7.38 & $2.425-11.307$ & 0.02 \\
Age & 0.992 & $0.569-1.451$ & 0.285 \\
Gender & 0.622 & $0.489-1.266$ & 0.531 \\
Fuhrman grade & 9.579 & $3.110-10.355$ & 0.103 \\
PTTG1 expression & 0.838 & $0.358-1.172$ & 0.027 \\
\hline
\end{tabular}

PTTG1, pituitary tumor-transforming gene-1.

compared to those with lower expression (PTTG1-). In the group of PTTG1+ patients, the mean and median survival time was 59.722 and 47 months, respectively; however, the mean and median survival time in the PTTG1- group was 88.285 and 79 months, respectively. The log-rank test revealed that the survival rates were significantly different between the two groups (log-rank; P=0.011, Fig. 4A). Similarly, patients without regional lymph node involvement (N0) exhibited a better prognosis compared to those with regional lymph node involvement (N+) (log-rank, $\mathrm{P}=0.024$; Fig. 4B).

In addition, the multivariate Cox regression analysis indicated that $\mathrm{PTTG}$ expression $(\mathrm{P}=0.027)$ and $\mathrm{N}$ stage $(\mathrm{P}=0.020)$ were independent prognostic factors for the overall survival of ccRCC patients (Table II).

\section{Discussion}

PTTG1 was isolated from rat pituitary tumor cells in 1997 and identified as a pituitary-derived transforming gene (7). Structural homology led to the identification of the PTTG1 protein as a vertebrate securin critical in regulating sister chromatid separation during mitosis (8). PTTG1 overexpression has been reported in a variety of endocrine-related tumors, particularly pituitary, thyroid, breast, ovarian and uterine tumors, as well as non-endocrine-related cancers involving the central nervous, pulmonary and gastrointestinal systems. PTTG1 levels were found to be correlated with tumor invasiveness (14) and PTTG1 has been identified as a key signature gene associated with tumor metastasis (10).

NIH3T3 cells (15) and human embryonic kidney 293 cells (16) stably transfected with PTTG1 exhibited increased cell proliferation rates compared to control vector-transfected cells. Studies in PC3 cells reported tetracycline-regulated PTTG1 expression and PTTG1-induced cell growth (17). Inhibition of cell proliferation by PTTG1 siRNA has been reported in M10 melanoma (18), HeLa S3 (19) and SH-J1 hepatoma cells (20).

Evidence suggests that an important transforming mechanism underlying PTTG1 overexpression involves the induction of chromosomal instability and aneuploidy. p53-deficient MG-63 osteosarcoma cells transiently or stably transfected with PTTG1-enhanced green fluorescent protein 
were investigated for signs of aneuploidy, such as the presence of micronuclei, macronuclei, or chromosomal bridges (21).

Bernal et al (22) reported that PTTG1 specifically interacts with p53 in vitro and in vivo and that this interaction blocks specific binding of p53 to DNA and inhibits its transcriptional activity. In PTTG1-deficient tumor cells, the proapoptotic and transactivation functions of p53 were potentiated. In addition, the overexpression of PTTG1 in hepatocellular carcinoma cell lines attenuated p53 induction of apoptosis (20). Thus, these results suggest a tumorigenic mechanism for PTTG1, as the inhibition of p53-mediated apoptosis by high securin expression may explain the survival of tumor cells harboring functional p53 (22). In other studies, siRNA-based techniques efficiently suppressed endogenous PTTG1 and inhibited cell proliferation in PC3 prostate cancer cells (17) and hepatocellular carcinoma cell lines $(20,23)$.

To the best of our knowledge, this is the first study to indicate the clinical significance of PTTG1 in ccRCC. RT-qPCR in 44 ccRCC and paired ADT samples demonstrated a significant increase of PTTG1 mRNA in the ccRCC samples. Further immunohistochemical analysis in the 44 paired ccRCC and ADT samples confirmed the overexpression of PTTG1 protein in the tumor tissues. These results indicate that PTTG1 may play a significant role in the initiation and progression of malignancies.

To further investigate the prognostic value of PTTG1, immunohistochemical analysis was performed to evaluate the correlation between PTTG1 expression and various clinicopathological parameters. In this study, we demonstrated that the increased PTTG1 expression was significantly correlated with tumor size, Fuhrman grade, stage, N classification, metastasis and recurrence. According to the Kaplan-Meier analysis, PTTG1 protein expression in ccRCC was significantly correlated with overall survival. Patients with high PTTG1 expression levels exhibited a shorter survival time compared to those with low PTTG1 levels. The log-rank test revealed that the PTTG1- group exhibited a more favorable prognosis compared to the PTTG1+ group. In addition, the TNM stage of ccRCC was found to be closely associated with prognosis (24). Consistent with those findings, in the present study, PTTG1 expression and N classification were independent prognostic factors for the overall survival of ccRCC patients in the multivariate Cox regression analysis. Therefore, this study revealed that there were significant correlations between the PTTG1 expression level and clinicopathological parameters and, therefore, PTTG1 may represent a potential prognostic marker and therapeutic target for ccRCC.

To the best of our knowledge, this is the first study aimed at evaluating the possibility of using PTTG1 as an indicator of disease progression in the clinical setting, as well as a prognostic marker for ccRCC patient survival. However, it should be noted that our study was a single hospital-based, retrospective study and further multi-centered or community-based prospective studies are required to verify our findings.

\section{Acknowledgements}

This study was supported by the National Natural Science Foundation of China (grant no. 81101524).

\section{References}

1. Siegel R, Ma J, Zou Z and Jemal A: Cancer statistics, 2014. CA Cancer J Clin 64: 9-29, 2014.

2. Singer EA, Gupta GN, Marchalik D and Srinivasan R: Evolving therapeutic targets in renal cell carcinoma. Curr Opin Oncol 25: 273-280, 2013.

3. Macher-Goeppinger S, Bermejo JL, Schirmacher P, Pahernik S, Hohenfellner M and Roth W: Senescence-associated protein p400 is a prognostic marker in renal cell carcinoma. Oncol Rep 30: 2245-2253, 2013.

4. Tanaka T, Kuramitsu Y, Wang Y, et al: Glyoxalase 1 as a candidate for indicating the metastatic potential of SN12C human renal cell carcinoma cell clones. Oncol Rep 30: 2365-2370, 2013.

5. Wei C, Lai YQ, Li XX and Ye JX: TGF- $\beta$-activated kinase-1: a potential prognostic marker for clear cell renal cell carcinoma. Asian Pac J Cancer Prev 14: 315-320, 2013.

6. Iwamoto H, Kanda Y, Sejima T, Osaki M, Okada F and Takenaka A: Serum miR-210 as a potential biomarker of early clear cell renal cell carcinoma. Int J Oncol 44: 53-58, 2014.

7. Pei L and Melmed S: Isolation and characterization of a pituitary tumor-transforming gene (PTTG). Mol Endocrinol 11: 433-441, 1997.

8. Zou H, McGarry TJ, Bernal T and Kirschner MW: Identification of a vertebrate sister-chromatid separation inhibitor involved in transformation and tumorigenesis. Science 285: 418-422, 1999.

9. Feng ZZ, Chen JW, Yang ZR, Lu GZ and Cai ZG: Expression of PTTG1 and PTEN in endometrial carcinoma: correlation with tumorigenesis and progression. Med Oncol 29: 304-310, 2012.

10. Yoon CH, Kim MJ, Lee H, et al: PTTG1 oncogene promotes tumor malignancy via epithelial to mesenchymal transition and expansion of cancer stem cell population. J Biol Chem 287: 19516-19527, 2012.

11. Fuhrman SA, Lasky LC and Limas C: Prognostic significance of morphologic parameters in renal cell carcinoma. Am J Surg Pathol 6: 655-663, 1982.

12. Wei C, Wu S, Li X, Wang Y, Ren R, Lai Y and Ye J: High expression of FER tyrosine kinase predicts poor prognosis in clear cell renal cell carcinoma. Oncol Lett 5: 473-478, 2013.

13. Livak KJ and Schmittgen TD: Analysis of relative gene expression data using real-time quantitative PCR and the $2^{-\Delta \Delta C}$ method. Methods 25: 402-408, 2001.

14. Demeure MJ, Coan KE, Grant CS, et al: PTTG1 overexpression in adrenocortical cancer is associated with poor survival and represents a potential therapeutic target. Surgery 154: 1405-1416, 2013.

15. Kakar SS and Jennes L: Molecular cloning and characterization of the tumor transforming gene (TUTR1): a novel gene in human tumorigenesis. Cytogenet Cell Genet 84: 211-216, 1999.

16. Hamid T, Malik MT and Kakar SS: Ectopic expression of PTTG1/securin promotes tumorigenesis in human embryonic kidney cells. Mol Cancer 4: 3, 2005.

17. Huang SQ, Liao QJ, Wang XW, Xin DQ, Chen SX, Wu QJ and Ye G: RNAi-mediated knockdown of pituitary tumor-transforming gene-1 (PTTG1) suppresses the proliferation and invasive potential of PC3 human prostate cancer cells. Braz J Med Biol Res 45: 995-1001, 2012.

18. Caporali S, Alvino E, Levati L, et al: Down-regulation of the PTTG1 proto-oncogene contributes to the melanoma suppressive effects of the cyclin-dependent kinase inhibitor PHA-848125. Biochem Pharmacol 84: 598-611, 2012.

19. Solbach C, Roller M, Peters S, Nicoletti M, Kaufmann M and Knecht R: Pituitary tumor-transforming gene (PTTG): a novel target for anti-tumor therapy. Anticancer Res 25: 121-125, 2005.

20. Cho-Rok J, Yoo J, Jang YJ, et al: Adenovirus-mediated transfer of siRNA against PTTG1 inhibits liver cancer cell growth in vitro and in vivo. Hepatology 43: 1042-1052, 2006.

21. Hsu YH, Liao LJ, Yu CH, et al: Overexpression of the pituitary tumor transforming gene induces p53-dependent senescence through activating DNA damage response pathway in normal human fibroblasts. J Biol Chem 285: 22630-22638, 2010.

22. Bernal JA, Luna R, Espina A, et al: Human securin interacts with p53 and modulates p53-mediated transcriptional activity and apoptosis. Nat Genet 32: 306-311, 2002.

23. Liang M, Chen X, Liu W, Li S, Li C, Jiang L and Lv S: Role of the pituitary tumor transforming gene 1 in the progression of hepatocellular carcinoma. Cancer Biol Ther 11: 337-345, 2011.

24. Levi F, Ferlay J, Galeone C, Lucchini F, Negri E, Boyle P and La Vecchia C: The changing pattern of kidney cancer incidence and mortality in Europe. BJU Int 101: 949-958, 2008. 